\title{
2D RANDOM DIRAC FERMIONS: LARGE $N$ APPROACH
}

\author{
D. Serban \\ Service de Physique Théorique, CE Saclay, F-91191 Gif-sur-Yvette, France \\ serban@spht.saclay.cea.fr
}

\begin{abstract}
We study the symmetry classes for the random Dirac fermions in 2 dimensions. We consider $N_{f}$ species of fermions, coupled by different types of disorder. We analyse the renormalisation group flow at the order of one loop. At $N_{f}$ large, the disorder distribution flows to an isotropic distribution and the effective action is a sigma model.
\end{abstract}

\section{Introduction}

Random Dirac fermions in 2 dimensions appear in various contexts in condensed matter physics. They were used to model disorder in systems with degenerate Fermi surface [2] , in the $d$-wave superconductors $[3,4]$ or to investigate the plateau transition in the integer quantum Hall effect [5]. In all these problems, the main question concerns the localization of quasiparticles, often different from the generic Anderson localization.

The universal properties of a system where randomness is present depend largely on the discrete symmetries, like time reversal invariance or spin rotation symmetry. This principle governs the Wigner-Dyson classification of random hermitian matrices [1]. Considering other discrete symmetries, like particle-hole or chiral symmetry, led Altland and Zirnbauer [6] to extend this classification. However, it was recognized that the physics of $2 \mathrm{~d}$ Dirac fermions is richer than expected from the random matrix classification, in the sense that for the same symmetry class, some systems may be critical and other not. One reason is that in two dimension, the sigma models, which are supposed to describe the universal physics, can support a topological term. In the form of a theta or WZW term, they can render the model critical [7, 8]. Sometimes, the presence of such a term can be deduced from general considerations $[7,8]$, in other situations it can be obtained exactly $[3,16]$. A possible strategy, adopted in $[14,15]$ and applied here to all classes, is to try to 
derive the effective sigma model, topological term included, by chain of controlled approximations. The drawback is that we have to consider a large number of fermion species $N_{f}$ in order to have a control parameter for the approximation. This means that the most interesting cases, where $N_{f}$ is small, are out of reach of this method.

The symmetry classes for $2 \mathrm{~d}$ random Dirac hamiltonians, were recently analysed [9]. The constraint that the discrete symmetries should also preserve the form of the Dirac operator leads to a refinement of the Altland-Zirnbauer classification, in which the classes AIII, $C$ I and DIII, appear each in two different forms. Here, we pursue the characterization of the symmetry classes for the Dirac Hamiltonians of [9]. We compute the beta function for all the disorder perturbations. When the number of fermion components $N_{f}$ is large the flow of the coupling constants generically closes on a single coupling constant, a fact which simplifies considerably the derivation of the effective action. This was done for classes $D$ in [14] and for classes $C$ and $C$ I in [15], and we refer to these papers for the details of the method. Without surprise, we obtain the sigma models defined on the manifolds identified by Zirnbauer [6] . The two different versions of classes $A$ III, $C$ I and $D$ III give rise to sigma models with or without a WZW term.

We consider random Dirac Hamiltonians in 2d, in the representation

$$
H=\left(\begin{array}{cc}
V+M & -2 i \partial_{z}+A_{\bar{z}} \\
-2 i \partial_{z}+A_{\bar{z}} & V-M
\end{array}\right),
$$

where $\partial_{z}=\left(\partial_{x}-i \partial_{y}\right) / 2, \partial_{\bar{z}}=\left(\partial_{x}+i \partial_{y}\right) / 2$ and $V, M, A_{z}$ and $A_{\bar{z}}$ are random matrix fields of dimension $N_{f}$.

The discrete symmetries preserving the form of the Dirac Hamiltonian are $[9]$

$$
\begin{aligned}
& H=-P H P^{-1}, \quad P=\left(\begin{array}{cc}
\gamma & 0 \\
0 & -\gamma
\end{array}\right), P P^{\dagger}=1, P^{2}=1 \\
& H=\epsilon_{c} C H^{T} C^{-1}, \quad C=\left(\begin{array}{cc}
0 & \sigma \\
-\epsilon_{c} \sigma & 0
\end{array}\right), C C^{\dagger}=1, C^{T}= \pm C .
\end{aligned}
$$

The first condition will be called chiral symmetry, while the second one could be called particle-hole symmetry when $\epsilon_{c}=-1$ and time reversal symmetry (or spin rotation invariance) when $\epsilon_{c}=+1$. The symmetry conditions impose constraints on the matrix fields $V, M, A_{z}$ and $A_{\bar{z}}$, which are listed in [9]. We consider gaussian probability distribution for the disorder, compatible with the symmetry conditions.

The spectral properties of the Hamiltonians 1 can be analysed using the supersymmetry [10] (or replica) method, in which products of Green functions are represented by gaussian integral over equal numbers of 
fermionic and bosonic Dirac fields. Average over gaussian randomness introduce effective coupling between the bosonic and fermionic fields, the coupling constants being the disorder variances.

The first step of our analysis is the renormalisation group flow for the effective coupling constants. The analysis can be eventually performed at all orders [11]. In the section 3 we give the one loop beta functions for all the clases and for any $N_{f}$. Generically, at large $N_{f}$ the flow closes on a single coupling constant, corresponding to equal variances for different types of disorder. ${ }^{1}$ In this case, the effective interaction, quartic in the the Dirac fields, can be decoupled via a Hubbard-Stratonovich transformation using a (super)matrix field $Q$ whose dimension does not depend on $N_{f}$. The number of components of the Dirac fields appear as an overall factor and it can be used as control parameter for a saddle point approximation.

The effective action for the field $Q$, hence the saddle point equation, has global invariance, described by a group $G$. The solutions of the saddle point equation break the invariance to a subgroup $H$, so that the manifold of saddle points is given by $G / H$. The low energy behaviour is obtained by taking slowly varying configurations of the field on $G / H$ (Goldstone modes) and evaluating the action for these modes. The result is a sigma model on some symmetric (super) space. Given the fact that in two dimensions sigma models can support a topological term, we may obtain a theta term or a WZW one. In the last section, we give a list of bilinears in Dirac fields to which couple the field $Q$, the invariance group $G$ of $Q$, as well as a diagonal solution of the saddle point equation $q_{0}$ together with its stabiliser $H$. Together with the coupling constants, these are the basic ingredients to characterize the sigma model for each symmetry class.

\section{Disorder perturbations}

We use notations similar to [14] . To obtain a compact notation, we introduce supermultiplets

$$
\phi=\left(\begin{array}{c}
\psi_{+} \\
\psi_{-}^{T} \\
\beta_{+} \\
\beta_{-}^{T}
\end{array}\right), \quad \phi^{t} \equiv\left(\psi_{-}, \psi_{+}^{T}, \beta_{-},-\beta_{+}^{T}\right)
$$

and similarly for $\bar{\phi}, \bar{\phi}^{t}$, where $\psi_{+}$and $\psi_{-}$are fermion multiplets with $N_{f}$ components while $\beta_{+}$and $\beta_{-}$are bosonic ghostswith the same number of components. The superscript $T$ denotes transposition. 
In the absence of disorder, the action for the Dirac fields is written

$$
S_{\mathrm{cft}}=\int \frac{d^{2} x}{2 \pi}\left(\phi^{t} \partial_{\bar{z}} \phi+\bar{\phi}^{t} \partial_{z} \bar{\phi}\right)
$$

and it corresponds to a conformal field theory with central charge $c=0$. From the holomorphic (antiholomorphic) fields $\phi(\bar{\phi})$, one can construct the current algebra $O S p\left(2 N_{f} \mid 2 N_{f}\right)_{k=1}[16]$.

The perturbation induced by disorder is

$$
S_{\text {pert }}=g_{i} \int \frac{d^{2} x}{2 \pi} \mathcal{O}_{i}
$$

where $g_{i}$ are the disorder variances associated to different disorder invariants and the operators $\mathcal{O}_{i}$ are defined as follows

$$
\begin{aligned}
& \mathcal{O}_{A}=\sum_{I \in \mathcal{A}}\left(\phi^{t} E_{I}^{-} \phi\right)\left(\bar{\phi}^{t} E_{I}^{-} \bar{\phi}\right), \quad \mathcal{O}_{a}=\left(\phi^{t} E_{0}^{-} \phi\right)\left(\bar{\phi}^{t} E_{0}^{-\bar{\phi}}\right), \\
& \mathcal{O}_{V}=\sum_{I \in \mathcal{V}}\left(\bar{\phi}^{t} E_{I}^{-} \phi\right)^{2}, \quad \mathcal{O}_{v}=\left(\bar{\phi}^{t} E_{0}^{-} \phi\right)^{2}, \\
& \mathcal{O}_{M}=\sum_{I \in \mathcal{M}}\left(\bar{\phi}^{t} E_{I}^{+} \phi\right)^{2}, \quad \mathcal{O}_{m}=\left(\bar{\phi}^{t} E_{0}^{+} \phi\right)^{2},
\end{aligned}
$$

where

$$
E_{I}^{ \pm}=\left(\begin{array}{cc}
e_{I} & 0 \\
0 & \pm e_{I}^{T}
\end{array}\right)
$$

$e_{I}$ are generators of $g l\left(N_{f}\right)$ normalized by $\operatorname{Tr}\left(e_{I} e_{J}\right)=\delta_{I J}$ and $\mathcal{A}, \mathcal{V}, \mathcal{M}$ are subsets of indices of $s l\left(N_{f}\right)$ which depend on the symmetry class. $e_{0}$ is the identity normalized by $\operatorname{Tr} e_{0}^{2}=1$.

\section{The one loop beta functions}

To study the renormalisation group flow for the coupling constants, we need the corresponding beta function. They can be derived from the operator product expansion of the perturbing operators $\mathcal{O}_{i}$

$$
\mathcal{O}_{i}(z, \bar{z}) \mathcal{O}_{j}(0) \simeq \frac{1}{z \bar{z}} C_{i j}^{k} \mathcal{O}_{k}(0)+\text { reg. }
$$

The one loop beta functions are given by [13]

$$
\beta_{k} \equiv l \partial_{l} g_{k}=-\sum_{i, j} C_{i j}^{k} g_{i} g_{j}
$$

The results for different classes are listed in the remaining of this section.

\section{The non-chiral classes}

Class $0(A)$ 
This class do not posses neither chiral invariance, nor particle-hole/ time reversal symmetry. We set $N_{f}=N$ and consider only disorder which do not break parity invariance of the action. The disorder fields $A$, $V$ and $M$ belong to $g l(N)$; the $\operatorname{sl}(N)$ part generates the perturbation operators $\mathcal{O}_{A}, \mathcal{O}_{V}, \mathcal{O}_{M}$, while the $g l(1)$ part generates $\mathcal{O}_{a}, \mathcal{O}_{v}, \mathcal{O}_{m}$. The corresponding beta functions are

$$
\begin{aligned}
\beta_{A} & =-\frac{D}{2} g_{A}^{2}-\frac{D}{8}\left(g_{M}^{2}+g_{V}^{2}\right)+\frac{B}{4} g_{M} g_{V}+\frac{1}{N} g_{m} g_{V}+\frac{1}{N} g_{M} g_{v} \\
\beta_{a} & =\frac{N y}{4} g_{M} g_{V}+\frac{1}{N} g_{m} g_{v}, \\
\beta_{M} & =-x g_{M}^{2}-\frac{1}{N}\left(g_{m}+g_{v}\right) g_{M}+\left(2 x-\frac{D}{2}\right) g_{A} g_{M}+\frac{B}{2} g_{A} g_{V} \\
& +\frac{2}{N} g_{A} g_{v}+\frac{2}{N} g_{a}\left(g_{V}+g_{M}\right)-x g_{M} g_{V}, \\
\beta_{V} & =x g_{V}^{2}+\frac{1}{N}\left(g_{m}+g_{v}\right) g_{V}+\left(2 x-\frac{D}{2}\right) g_{A} g_{V}+\frac{B}{2} g_{A} g_{M} \\
& +\frac{2}{N} g_{A} g_{m}+\frac{2}{N} g_{a}\left(g_{M}+g_{V}\right)+x g_{M} g_{V}, \\
\beta_{m} & =-\frac{1}{N} g_{m}\left(g_{m}+g_{v}\right)-C g_{m}\left(g_{M}+g_{V}\right)+2 C g_{A} g_{m} \\
& +\frac{2}{N} g_{a}\left(g_{m}+g_{v}\right)+\frac{N y}{2} g_{A} g_{V}, \\
\beta_{v} & =\frac{1}{N} g_{v}\left(g_{v}+g_{m}\right)+C g_{v}\left(g_{V}+g_{M}\right)+2 C g_{A} g_{v} \\
& +\frac{2}{N} g_{a}\left(g_{v}+g_{m}\right)+\frac{N y}{2} g_{A} g_{M},
\end{aligned}
$$

where the constants, with group theoretical significance, are

$$
\begin{aligned}
D & =-2 N, \quad C=\frac{N^{2}-1}{N}, \quad B=\frac{2\left(N^{2}-4\right)}{N}, \\
x & =-\frac{1}{N}, \quad y=\frac{4\left(N^{2}-1\right)}{N^{2}} .
\end{aligned}
$$

In the large $N$ limit, the line $g_{A}=g_{V}=g_{M}=g_{m}=-g_{v}=2 g_{a}=$ $g \sim 1 / N$ is preserved by the RG flow. It is interesting to note that we cannot obtain an invariant line with all the coupling constants positive.

Classes $3_{-}$and $4_{-}(D$ and $C)$

These classes have particle-hole symmetry $\left(\epsilon_{c}=-1\right)$, inforced by symmetric, respectively antisymmetric matrix $\sigma^{T}= \pm \sigma$. We set $N_{f}=$ $2 N$, but for class $D, N$ can be half integer. The random fields $A$ and $V$ belong to the algebra $s o(2 N)$ (case $D$ ) and $s p(2 N)$ (class $C$ ). These algebra are spanned by a subset of the $s l(2 N)$ generators $e_{I}, \tau_{I}$, selected by the condition $\tau_{I}=-\sigma \tau_{I}^{T} \sigma^{-1}$. The field $M$ belongs to the complement of these algebras with respect to $\operatorname{sl}(2 N)$, spanned by $T_{I}$ with $T_{I}=$ 
$\sigma T_{I}^{T} \sigma^{-1}$. Operators $\mathcal{O}_{a}, \mathcal{O}_{v}$ are not allowed by these symmetry classes. The beta functions are

$$
\begin{aligned}
\beta_{A} & =-\frac{D_{\tau \tau}}{2} g_{A}^{2}-\frac{D_{T T}}{8} g_{M}^{2}-\frac{D_{\tau \tau}}{8} g_{V}^{2}+\frac{B_{\tau T}}{4} g_{M} g_{V}+\frac{1}{2 N} g_{m} g_{V} ; \\
\beta_{M} & =-x_{T T T} g_{M}^{2}-\frac{1}{2 N} g_{m} g_{M}+\left(2 x_{\tau T \tau}-\frac{D_{\tau T}}{2}\right) g_{A} g_{M}+\frac{B_{\tau \tau}}{2} g_{A} g_{V} \\
& -x_{\tau T \tau} g_{M} g_{V}, \\
\beta_{V} & =x_{\tau \tau \tau} g_{V}^{2}+\frac{1}{2 N} g_{m} g_{V}+\left(2 x_{\tau \tau \tau}-\frac{D_{\tau \tau}}{2}\right) g_{A} g_{V}+\frac{B_{\tau T}}{2} g_{A} g_{M} \\
& +\frac{1}{N} g_{m} g_{A}+x_{T \tau T} g_{M} g_{V}, \\
\beta_{m} & =-\frac{1}{2 N} g_{m}^{2}+2 C g_{A} g_{m}+N y_{\tau \tau} g_{A} g_{V}-C g_{V} g_{m}-C^{\prime} g_{m} g_{M},
\end{aligned}
$$

with the group coefficients (again, the upper sign is for class $D$ and the lower one for class $C$ ) :

$$
\begin{aligned}
C & =\frac{2 N \mp 1}{2}, \quad C^{\prime}=C_{s l(2 N)}-C=\frac{(2 N \mp 1)(N \pm 1)}{2 N} \\
D_{\tau \tau} & =-2(N \mp 1), \quad D_{\tau T}=-2 N, \quad D_{T T}=-2(N \pm 1) \\
x_{\tau \tau \tau} & =-x_{\tau T \tau}= \pm \frac{1}{2}, \quad x_{T T T}=-\frac{1 \mp N}{2 N}, \quad x_{T \tau T}=\frac{1 \pm N}{2 N} \\
y_{\tau \tau} & =\frac{(2 N \mp 1)}{N}, \quad B_{\tau \tau}=2(N \mp 1) \\
B_{\tau T} & =2 \frac{N^{2}-1}{N}, \quad B_{T T}=2 \frac{(N \pm 2)(N \mp 1)}{N} .
\end{aligned}
$$

At large $N$, an invariant line is given by $g_{A}=g_{M}=g_{V}=g_{m}=g$.

Classes $3_{+}$and $4_{+}(A I I$ and $A I)$

These classes have time reversal (spin rotation) symmetry, with $\epsilon_{c}=1$ and $\sigma^{T}= \pm \sigma$. They are similar to classes $3_{-}$and $4_{-}$, with the random fields $V$ and $M$ changing roles: now $M$ belongs to $s o(2 N)$ and $s p(2 N)$ respectively, while $V$ belongs to the complement with respect to $\operatorname{sl}(2 N)$. The beta functions can be obtained from that of the classes $3_{-}$and $4_{-}$ by changing $g_{M} \leftrightarrow-g_{V}$ and $g_{m} \leftrightarrow-g_{v}$.

\section{The chiral classes}

- The first type of chiral classes corresponds to $\gamma=1$ in eq. 2 and it comprises three classes. Only disorder of the vector potential type is allowed, and these classes can be seen as limits of the previous ones when $g_{V}, g_{M}, g_{v}, g_{m} \rightarrow 0$.

Class 1 (AIII)

The random gauge potential $A$ belongs to $g l(N) \simeq g l(1) \otimes \operatorname{sl}(N)$ $\left(N_{f}=N\right)$.

$$
\beta_{A}=-\frac{D}{2} g_{A}^{2}, \quad \beta_{a}=0,
$$


with $D=-2 N$.

Classes 5 and 6 (DIII and $C I)$

In addition to the chiral symmetry, these classes posses particle-hole symmetry. We set $N_{f}=2 N$. The random gauge potential $A$ belongs to $s o(2 N)$ and $s p(2 N)$ respectively

$$
\beta_{A}=-\frac{D_{\tau \tau}}{2} g_{A}^{2}
$$

with $D_{\tau \tau}=-2(N \mp 1)$.

- The second type of chiral classes correspond to $\gamma=\sigma_{3}$ and it comprises five classes. We set $N_{f}=2 N$, but for classes 8 and $9_{+} N$ should be understood as even integer.

Class $2(A I I I)$

The chiral symmetry imposes the constraint $[A, \gamma]=0$ to the random gauge potential; this condition selects an algebra $g l(N) \otimes g l(N) \simeq$ $(g l(1) \otimes s l(N))^{\otimes 2}$ out of $g l(2 N)$. The two $g l(1)$ parts are generated by the identity and $\gamma$ and we denote the associated coupling constants by $g_{a}$ and $g_{a-}$. We denote by $e_{i}$ the generators of $s l(N) \otimes s l(N)$. The two $\operatorname{sl}(N)$ components can have different variances, $g_{A 1}$ and $g_{A 2}$, with $g_{A}^{ \pm}=\left(g_{A 1} \pm g_{A 2}\right) / 2$.

The fields $V$ and $M$ are constrained by $\{V, \gamma\}=0,\{M, \gamma\}=0$, so they belong to $g l(2 N) \backslash(g l(N) \otimes g l(N))$, generated by $e_{\alpha}$. Operators $\mathcal{O}_{v}$ and $\mathcal{O}_{m}$ are not allowed by the chiral classes.

The beta functions are

$$
\begin{aligned}
& \beta_{A}^{+}=-\frac{D_{i i}}{2}\left(g_{A}^{+2}+g_{A}^{-2}\right)-\frac{D_{\alpha \alpha}}{8}\left(g_{M}^{2}+g_{V}^{2}\right)+\frac{B_{\alpha \alpha}}{4} g_{M} g_{V}, \\
& \beta_{A}^{-}=-D_{i i} g_{A}^{+} g_{A}^{-}, \quad \beta_{a}=\frac{N y_{\alpha}}{2} g_{M} g_{V}, \quad \beta_{a-}=-\frac{D_{\alpha \alpha}}{8}\left(g_{M}^{2}+g_{V}^{2}\right), \\
& \beta_{M}=-x_{\alpha \alpha \alpha} g_{M}\left(g_{M}+g_{V}\right)-\frac{D_{i \alpha}}{2} g_{A}^{+} g_{M}+\frac{B_{i \alpha}}{2} g_{A}^{+} g_{V}+\frac{1}{N} g_{a}\left(g_{M}+g_{V}\right), \\
& \beta_{V}=x_{\alpha \alpha \alpha} g_{V}\left(g_{V}+g_{M}\right)-\frac{D_{i \alpha}}{2} g_{A}^{+} g_{V}+\frac{B_{i \alpha}}{2} g_{A}^{+} g_{M}+\frac{1}{N} g_{a}\left(g_{V}+g_{M}\right) .
\end{aligned}
$$

with the group coefficients

$$
\begin{aligned}
D_{i i} & =D_{s l(N)}=-2 N, \quad D_{\alpha \alpha}=D_{g l(2 N)}-D_{g l(N)}=-2 N, \\
D_{i \alpha} & =D_{g l(2 N)} / 2+\frac{2}{N}=-2 N+\frac{2}{N}, \quad y_{\alpha}=2, \\
x_{\alpha \alpha \alpha} & =0, \quad B_{i \alpha}=\frac{2\left(N^{2}-1\right)}{N}, \quad B_{\alpha \alpha}=2 N .
\end{aligned}
$$

At large $N$, the line $g_{A}^{-}=0, g_{A}^{+}=g_{M}=g_{V}=2 g_{a}=4 g_{a-}$ is left invariant by the RG flow.

Classes 7 and $8(D I I I$ and $C I)$ 
These classes have both chiral symmetry and particle-hole symmetry, with the matrices inforcing these symmetries commuting.

We split the generators of $g l(2 N)$ in four groups $\tau_{i}, \tau_{\alpha}, T_{i}, T_{\alpha}$, with $\tau_{i}, T_{i}$ commuting with $\sigma_{3}$ and $\tau_{\alpha}, T_{\alpha}$ anticommuting with it; also, $\tau_{i, \alpha}=-\sigma \tau_{i, \alpha}^{T} \sigma^{-1}$ and $T_{i, \alpha}=\sigma T_{i, \alpha}^{T} \sigma^{-1}$. The random gauge potential $A$ is an element of $s o(N) \otimes s o(N)$ or $s p(N) \otimes s p(N)$ generated by $\tau_{i}$. If we choose the representation $\epsilon_{c}=-1, V$ belongs to $o(2 N) \backslash o(N) \otimes o(N)$ or $s p(2 N) \backslash s p(N) \otimes s p(N)$ respectively, generated by $\tau_{\alpha}$, while the field $M$ is generated by $T_{\alpha}$.

The beta functions are

$$
\begin{aligned}
& \beta_{A}^{-}=-D_{\tau \tau}^{i i} g_{A}^{+} g_{A}^{-} \\
& \beta_{A}^{+}=-\frac{D_{\tau \tau}^{i i}}{2}\left(g_{A}^{+2}+g_{A}^{-2}\right)-\frac{D_{T T}^{\alpha \alpha}}{8} g_{M}^{2}-\frac{D_{\tau \tau}^{\alpha \alpha}}{8} g_{V}^{2}+\frac{B_{\tau T}^{\alpha \alpha}}{4} g_{M} g_{V}, \\
& \beta_{M}=-x_{T T T}^{\alpha \alpha \alpha} g_{M}^{2}+\left(2 x_{\tau T \tau}^{i \alpha i}-\frac{D_{\tau T}^{i \alpha}}{2}\right) g_{A}^{+} g_{M}+\frac{B_{\tau \tau}^{i \alpha}}{2} g_{A}^{+} g_{V}-x_{\tau T \tau}^{\alpha \alpha \alpha} g_{M} g_{V}, \\
& \beta_{V}=x_{\tau \tau \tau}^{\alpha \alpha \alpha} g_{V}^{2}+\left(2 x_{\tau \tau \tau}^{i \alpha i}-\frac{D_{\tau \tau}^{i \alpha}}{2}\right) g_{A}^{+} g_{V}+\frac{B_{\tau T}^{i \alpha}}{2} g_{A}^{+} g_{M}+x_{T \tau T}^{\alpha \alpha \alpha} g_{M} g_{V},
\end{aligned}
$$

where the group coefficients are

$$
\begin{aligned}
D_{\tau \tau}^{i i} & =-N \pm 2, \quad D_{\tau \tau}^{\alpha \alpha}=D_{T T}^{\alpha \alpha}=-N, \\
D_{\tau \tau}^{i \alpha} & =D_{\tau T}^{i \alpha}=-N \pm 1 \\
x_{T T T}^{\alpha \alpha \alpha} & =x_{\tau \tau \tau}^{\alpha \alpha \alpha}=-x_{\tau T \tau}^{\alpha \alpha \alpha}=-x_{T \tau T}^{\alpha \alpha \alpha}= \pm \frac{1}{2}, \quad x_{\tau T \tau}^{i \alpha i}=x_{\tau \tau \tau}^{i \alpha i}=0 \\
B_{\tau T}^{\alpha \alpha} & =N, \quad B_{\tau \tau}^{i \alpha}=B_{\tau T}^{i \alpha}=N \mp 1 .
\end{aligned}
$$

In the large $N$ limit the RG flow preserve the line $g_{A}^{-}=0, g_{A}^{+}=g_{M}=g_{V}$.

Classes $9_{+}$and $9_{-}(D \mathrm{I}$ and $C \mathrm{II})$

These classes have also both particle-hole and chiral symmetry, with the matrices enforcing these constraints anticommuting, $\{\gamma, \sigma\}=0$.

If we choose $\epsilon_{c}=-1$, the matrix $\sigma$ defining the particle-hole symmetry is symmetric for class $D I$ and antisymmetric for class $C$ II, as for the cases $3_{-}$and $4_{-}$or 7 and 8 . The opposite choice exchanges the symmetry of the matrix $\sigma$ between the two classes.

As in the previous case, we split the $g l(2 N)$ generators into four classes. Since $\{\gamma, \sigma\}=0, \tau_{i}$ spans now $g l(N)$, to which the field $A$ belong. $V$ is generated by $\tau_{\alpha}$, which span $o(2 N) \backslash g l(N)$ and $s p(2 N) \backslash g l(N)$ respectively. $M$ is again generated by $T_{\alpha}$.

$$
\begin{aligned}
\beta_{a-} & =-\frac{D_{T T}^{\alpha \alpha}}{8} g_{M}^{2}-\frac{D_{\tau \tau}^{\alpha \alpha}}{8} g_{V}^{2}, \quad \beta_{A}^{-}=-D_{\tau \tau}^{i i} g_{A}^{+} g_{A}^{-} \\
\beta_{A}^{+} & =-\frac{D_{\tau \tau}^{i i}}{2}\left(g_{A}^{+2}+g_{A}^{-2}\right)-\frac{D_{T T}^{\alpha \alpha}}{8} g_{M}^{2}-\frac{D_{\tau \tau}^{\alpha \alpha}}{8} g_{V}^{2}+\frac{B_{\tau T}^{\alpha \alpha}}{4} g_{M} g_{V}
\end{aligned}
$$




$$
\begin{aligned}
\beta_{M} & =-x_{T T T}^{\alpha \alpha \alpha} g_{M}^{2}+\left(2 x_{\tau T \tau}^{i \alpha i}-\frac{D_{\tau T}^{i \alpha}}{2}\right) g_{A}^{+} g_{M}+\frac{B_{\tau \tau}^{i \alpha}}{2} g_{A}^{+} g_{V}-x_{\tau T \tau}^{\alpha \alpha \alpha} g_{M} g_{V}, \\
\beta_{V} & =x_{\tau \tau \tau}^{\alpha \alpha \alpha} g_{V}^{2}+\left(2 x_{\tau \tau \tau}^{i \alpha i}-\frac{D_{\tau \tau}^{i \alpha}}{2}\right) g_{A}^{+} g_{V}+\frac{B_{\tau T}^{i \alpha}}{2} g_{A}^{+} g_{M}+x_{T \tau T}^{\alpha \alpha \alpha} g_{M} g_{V},
\end{aligned}
$$

with

$$
\begin{aligned}
D_{\tau \tau}^{i i} & =-N, \quad D_{\tau \tau}^{\alpha \alpha}=-N \pm 2, \quad D_{T T}^{\alpha \alpha}=-N \mp 2 \\
D_{\tau \tau}^{i \alpha} & =-\frac{(N \pm 1)(N \mp 2)}{N}, \quad D_{\tau T}^{i \alpha}=-\frac{(N \mp 1)(N \pm 2)}{N}, \\
x_{T T T}^{\alpha \alpha \alpha} & =x_{\tau \tau \tau}^{\alpha \alpha \alpha}=x_{\tau T \tau}^{\alpha \alpha \alpha}=x_{T \tau T}^{\alpha \alpha \alpha}=0, \quad x_{\tau T \tau}^{i \alpha i}=\frac{1}{2 N} \mp \frac{1}{2} \\
x_{\tau \tau \tau}^{i \alpha i} & =\frac{1}{2 N} \pm \frac{1}{2}, \quad B_{\tau T}^{\alpha \alpha}=N, \quad B_{\tau \tau}^{i \alpha}=N \mp 1, \quad B_{\tau T}^{i \alpha}=N \pm 1 .
\end{aligned}
$$

At large $N$ the RG flow preserves the line $g_{A}^{-}=0$ and $g_{A}^{+}=g_{M}=$ $g_{V}=4 g_{a-}$.

We note that the equations listed above have interesting properties under the transformation $g_{M} \leftrightarrow-g_{V}$ and $g_{m} \leftrightarrow-g_{v}$. This operation leave the equations for classes $0,2,7$ and 8 invariant and exchanges the ones for classes $3_{ \pm}, 4_{ \pm}$and $9_{ \pm}$, therefore it is equivalent to changing the sign of $\epsilon_{c}$, when defined ${ }^{2}$. Moreover, on the line $g_{M}=-g_{V}=g$ ( $=g_{m}=-g_{v}$, when they exist), the flow for $g$ and the flow for $g_{A}$ decouple. This was noted in [18] for class 0 and was related to "spincharge" separation, for class $C$ in [12] .

\section{Hubbard-Stratonovich transformation and the sigma model}

We consider products of $n$ Green functions, $n_{R}$ retarded and $n_{A}$ advanced, and we need to introduce a copy of the multiplets 4 for each Green function. When the Hamiltonian has chiral or particle-hole invariance, the spectrum is symmetric with respect to $E=0$ and we do not need to distinguish between retarded and advanced sector. In this case, the density of states can be singular at the point $E=0$.

As already mentioned, when the coupling constant have equal values, the decoupling of the quartic interaction is particularly simple. At that particular point, the disorder perturbation can be written only in terms of singlets of $g l\left(N_{f}\right)$. The decoupling matrix $Q$ couples to these singlets and it has a size independent of $N_{f}$. Up to an additive constant, the effective action for $Q$ is

$$
S[Q]=-N_{f}\left[\frac{1}{\tilde{g}} \int \frac{d^{2} x}{2 \pi} \mathrm{S} \operatorname{Tr} Q^{2}-\mathbf{S} \operatorname{Tr} \ln \left(\begin{array}{ll}
Q & \partial \\
\bar{\partial} & Q
\end{array}\right),\right]
$$


where $\tilde{g} \sim N_{f} g$. Part of the invariance of the free action $5, O S p\left(2 n N_{f} \mid 2 n N_{f}\right)$, survives in the effective action 12 . We denote the residual invariance group by $G$.

The saddle point equation $\delta S[Q] / \delta Q=0$ can be solved making a diagonal ansaz $Q_{0}=\mu q_{0}$, where $q_{0}^{2}=1$ and $\mu$ has the dimension of a mass. As there is no scale in the problem, $g_{i}$ being dimensionless, $\mu$ is dynamically generated. The group $G$ acts on $Q$ by conjugation, and from a particular saddle point solution $q_{0}$ one generates a whole manifold by $q_{0} \rightarrow q=g q_{0} g^{-1}$. This manifold is isomorphic to $G / H$, where $H$ is the stabilizer of $q_{0}$. The last step in deriving the sigma model is to evaluate the action 12 on slowly varying configurations $q(x)$ on $G / H$. An elegant way of doing it is to use non-abelian bosonisation [17], as in $[4,14]$ and to write the free action as a WZW model. The field $q(x)$ becomes a mass term for the WZW field, which is forced to follow it. At scales larger than $\mu^{-1}$, the effective action is

$$
S[q]=-\frac{1}{16 \pi f} \int \frac{d^{2} x}{2 \pi} \operatorname{STr} \partial_{\nu} q \partial_{\nu} q+S_{\text {top }}[q]
$$

In the following, we give the main characteristics of the sigma model manifold for the 13 classes.

Class 0 ( $A$

$$
\begin{aligned}
& Q \sim \operatorname{Tr}_{\mathrm{gl}(\mathrm{N})}\left[\Sigma_{3}, \phi \bar{\phi}^{t}+\bar{\phi} \phi^{t}\right], \quad q_{0}=\Sigma_{3} \otimes \Lambda \\
& G=G L(n \mid n), \quad H=G L\left(n_{R} \mid n_{R}\right) \otimes G L\left(n_{A} \mid n_{A}\right),
\end{aligned}
$$

with $\Lambda=\operatorname{diag}\left(1_{n_{R}},-1_{n_{A}}\right)$.

Classes $3_{-}$and $4_{-}(D$ and $C)$

$$
\begin{aligned}
& Q \sim \operatorname{Tr}_{\mathrm{gl}(2 \mathrm{~N})} \Sigma\left(\phi \bar{\phi}^{t}+\bar{\phi} \phi^{t}\right) \Sigma^{-1}, \quad q_{0}=\Sigma_{3}, \\
& G=O S p(2 n \mid 2 n), \quad H=G L(n \mid n),
\end{aligned}
$$

with $\Sigma \equiv \operatorname{diag}(1, \sigma)$

Classes $3_{+}$and $4_{+}(A \mathrm{II}$ and $A \mathrm{I})$

$$
\begin{aligned}
& Q \sim \operatorname{Tr}_{\mathrm{gl}(2 \mathrm{~N})} \Sigma\left(\Sigma_{3} \phi \bar{\phi}^{t}+\bar{\phi} \phi^{t} \Sigma_{3}\right) \Sigma^{-1}, \quad q_{0}=\Lambda \\
& G=\operatorname{OSp}(2 n \mid 2 n), \quad H=\operatorname{OSp}\left(n_{R} \mid n_{R}\right) \otimes O S p\left(n_{A} \mid n_{A}\right) .
\end{aligned}
$$

- The effective action for the classes 1,5 and 6 is invariant under multiplication at left/right by holomorphic/antiholomorphic group elements. This is a sign that the theory is conformally invariant and indeed, when deriving the sigma model we obtain a WZW term.

Class 1 (AIII)

$$
Q \sim \operatorname{Tr}_{\mathrm{gl}(\mathrm{N})}\left[\Sigma_{3}, \phi \bar{\phi}^{t}\right], \quad \bar{Q} \sim 1 / 2 \operatorname{Tr}_{\mathrm{gl}(\mathrm{N})}\left[\Sigma_{3}, \bar{\phi} \phi^{t}\right],
$$




$$
q_{0}=\Sigma_{3} \otimes \Lambda, \quad G=G L(n \mid n)_{L} \otimes G L(n \mid n)_{R}, \quad H=G L(n \mid n) .
$$

The level of the WZW action is $k=N_{f}$.

Classes 5 and 6 (DIII and $C \mathrm{I}$ )

$$
\begin{aligned}
& Q \sim \operatorname{Tr}_{\mathrm{gl}(2 \mathrm{~N})}\left(\Sigma \phi \bar{\phi}^{t} \Sigma^{-1}\right), \quad \bar{Q} \sim \operatorname{Tr}_{\mathrm{gl}(2 \mathrm{~N})}\left(\Sigma \bar{\phi} \phi^{t} \Sigma^{-1}\right), \\
& q_{0}=\Sigma_{3}, \quad G=O S p(n \mid n)_{L} \otimes O S p(n \mid n)_{R}, \quad H=O S p(n \mid n) .
\end{aligned}
$$

The level of the WZW action is $k= \pm 2 N$, the change of sign indicating that the compact and non-compact sector switch from the orthogonal to the symplectic sector from one class to another.

- To take into account the chiral symmetry for the last five classes, we double the supermultiplets by

$$
\begin{aligned}
& \phi \quad \rightarrow \Phi=\frac{1}{\sqrt{2}}\left(\begin{array}{c}
\phi \\
\gamma \phi
\end{array}\right), \quad \phi^{t} \rightarrow \Phi^{t}=\frac{1}{\sqrt{2}}\left(\begin{array}{ll}
\phi^{t} & \phi^{t} \gamma
\end{array}\right), \\
& \bar{\phi} \quad \rightarrow \quad \bar{\Phi}=\frac{1}{\sqrt{2}}\left(\begin{array}{c}
\bar{\phi} \\
-\gamma \bar{\phi}
\end{array}\right), \quad \bar{\phi}^{t} \rightarrow \bar{\Phi}^{t}=\frac{1}{\sqrt{2}}\left(\begin{array}{ll}
\bar{\phi}^{t} & -\bar{\phi}^{t} \gamma
\end{array}\right)
\end{aligned}
$$

Class 2 (AIII)

$$
\begin{aligned}
& Q \sim \operatorname{Tr}_{\mathrm{gl}(2 \mathrm{~N})}\left[\Sigma_{3}, \Phi \bar{\Phi}^{t}+\bar{\Phi} \Phi^{t}\right], \quad q_{0}=\Sigma_{3} \otimes \sigma_{3} \\
& G=G L(n \mid n) \otimes G L(n \mid n), \quad H=G L(n \mid n)
\end{aligned}
$$

where $\sigma_{3}$ is the Pauli matrix acting in the extra space introduced to take into account the chiral symmetry. $G$ is selected from $G L(2 n \mid 2 n)$ by the condition $\left[g, \sigma_{1}\right]=0$.

Classes 7 and 8 (DIII and $C \mathrm{I})$

$$
\begin{aligned}
Q & \sim \operatorname{Tr}_{\mathrm{gl}(2 \mathrm{~N})} \Sigma\left(\Phi \bar{\Phi}^{t}+\bar{\Phi} \Phi^{t}\right) \Sigma^{-1}, \quad q_{0}=\Sigma_{3} \otimes \sigma_{3}, \\
G & =O S p(2 n \mid 2 n) \otimes O S p(2 n \mid 2 n), \quad H=O S p(2 n \mid 2 n) .
\end{aligned}
$$

$G$ is selected from $O S p(4 n \mid 4 n)$ by the condition $\left[g, \sigma_{1}\right]=0$

Classes $9_{-}$and $9_{+}(D \mathrm{I}$ and $C \mathrm{II})$

$$
\begin{aligned}
Q & \sim \operatorname{Tr}_{\mathrm{gl}(2 \mathrm{~N})} \Sigma\left(\Phi \bar{\Phi}^{t}+\bar{\Phi} \Phi^{t}\right) \Sigma^{-1}, \quad q_{0}=\Sigma_{3} \otimes \sigma_{3}, \\
G & =G L(2 n \mid 2 n), \quad H=O S p(2 n \mid 2 n) .
\end{aligned}
$$

The group $G$ is selected by from $O S p(4 n \mid 4 n)$ by $\left[g, \Sigma_{3} \otimes \sigma_{1}\right]$.

The classes $2,7,8$ and $9_{ \pm}$do not allow a theta term; also, there is no WZW term. Note that the sigma model is defined on the same manifold for the pair of classes 1 and 2 (class AIII), 5 and 7 (class DIII) and 6 and 8 (class $C \mathrm{I}$ ); the difference between the two members of a pair consists only on the presence or absence of the WZW term.

The coupling constant for the sigma model is given by the inverse of the number of the Dirac fermions, $f=1 / N_{f}$. 


\section{Notes}

1. Exceptions may be the coupling constants corresponding to some $u(1)$ part of the disorder, specific to classes $0,1,2$ and $9_{ \pm}$. This situation was analysed in the case 1 (AIII) in [4] and in [18] in a non-hermitian version of case 0 . For the moment, we ignore this point and analyse the case of equal disorder variances.

2. We thank to D. Bernard for pointing out that this can be realized on the Dirac Hamiltonian via a non-unitary transformation.

\section{References}

[1] F. Dyson, J. Math. Phys. 3 (1962) 140;

[2] F. Fradkin, Phys. Rev. B 33 3257;

[3] A.A. Nersesyan, A.M. Tsvelik and F. Wegner, Phys. Rev. Lett. 72 (1994) 2628;

[4] A. Altland, B.D. Simons, M.R. Zirnbauer, condmat/0006362;

[5] A.W.W. Ludwig, M.P.A. Fisher, R. Shankar, and G. Grinstein, Phys. Rev. B 50 (1994) 7526

[6] A. Altland and M. Zirnbauer, Phys. Rev. B 55 (1997) 1142; M. Zirnbauer, J. Math. Phys. 37 (1996) 4986;

[7] N. Read and D. Green, Phys. Rev B 61 (2000) 10267; T. Senthil and M.P.A. Fisher, Phys. Rev. B 61

[8] P. Fendley and R.M. Konik, Phys. Rev. B 62 (2000) 9359,

[9] D. Bernard and A. LeClair, "A classification of $2 \mathrm{~d}$ random Dirac fermions", cond-mat/0109552.

[10] K.B. Efetov, Adv. Phys. 32 (1983) 53;

[11] B. Gerganov, A. LeClair and M. Moriconi, hep-th/0011189;

[12] D. Bernard and A. LeClair, Phys. Rev. B 64 (2001), 045306.

[13] J. Cardy, in Les Houches, Eds. E. Brézin and J. Zinn-Justin, North-Holland, 1998;

[14] M. Bocquet, D. Serban, M.R. Zirnbauer, Nucl. Phys. B 578 (2000) 628;

[15] D. Bernard, N. Regnault, D. Serban, Nucl. Phys. B 612 (2001) 291;

[16] D. Bernard, "(Perturbed) conformal field theory applied to 2d disordered systems: an introduction", hep-th/9509137

[17] E. Witten, Commun. Math. Phys. 92 (1984) 455;

[18] S. Guruswamy, A. LeClair and A.W.W Ludwig, Nucl. Phys. B 583 (2000) 475; 